Over-expression of miRNA-125a could reduce serum autoantibodies and suppress autoimmune reaction in lpr mice.

\section{CORRELATION OF URINARY BIOMARKERS MCP1 AND NGAL WITH LUPUS NEPHRITIS (LN) HISTOLOGY AND DISEASE ACTIVITY SCORE}

R Ramamoorthy*, S Rajeswari, T Tamilselvam, M Kumudha. Madras Medical College, rheumatology, Chennai, India

\subsection{6/lupus-2017-000215.309}

Background and aims Treatment of $\mathrm{LN}$ requires an accurate assessment of disease activity.

This study aims to assess the correlation of urinary biomarkers MCP1 and NGAL with the disease activity in LN.

Methods Materials and Methods: Prospective study conducted in a tertiary care centre. 60 patients with SLE were recruited and divided into 3 groups: one with Active LN $(n=22)$, another with Inactive $\mathrm{LN}(\mathrm{n}=20)$ and third formed of SLE patients with no renal involvement $(n=18)$. Active $\mathrm{LN}$ patients underwent renal biopsy. For comparison another group of age and sex matched controls was taken $(n=20)$. Disease activity was correlated with biopsy and baseline characteristics. Urinary MCP1 and NGAL were measured and its correlation with disease activity was analysed.

Results In patients with active LN, both UMCP1/Cr and UNGAL/Cr were significantly elevated (92.78, $76.11 \mathrm{pg} / \mathrm{ml}$, $\mathrm{p}<0.001)$. In both control group and SLE without renal involvement the values of UMCP1/Cr and UNGAL/Cr were normal $(24.44,22.22 \mathrm{pg} / \mathrm{ml}$ in control and 24.3, $22.80 \mathrm{pg} / \mathrm{ml}$ in SLE without renal involvement). In patients with inactive $\mathrm{LN}$ the values of $\mathrm{UMCP} 1 / \mathrm{Cr}$ and UNGAL/Cr were observed to be significantly higher than control $(44.18,38.45 \mathrm{pg} / \mathrm{ml}$, $\mathrm{p}<0.005)$ and lower than those of active LN. Values of $\mathrm{UMCP} 1 / \mathrm{Cr}$ and UNGAL/Cr were found to be in close correlation with mean rSLEDAI scores of active LN (10) and inactive $\mathrm{LN}(3.6)$ and disease activity as per histopathology.

Conclusions Levels of urinary biomarkers UMCP1 and UNGAL were significantly elevated in active LN and found to have excellent correlation with histopathological disease activity index and rSLEDAI scores.

\section{STUDY ON THE ROLE AND MECHANISMS OF ABERRANT EXPRESSION OF TRANSCRIPTION FACTOR WT1 IN THE PSORIASIS VULGARIS LESION FORMATION}

W Ruifang*, Z Ming, S Yuwen, L Qianjin. The Second Xiangya Hospital of Central South University, Dermatology, Chang Sha, China

\subsection{6/lupus-2017-000215.310}

Background and aims Psoriasis vulgaris (PV) is a chronic inflammatory skin disease characterised by abnormal keratinocytes proliferation and apoptosis. Evidence has showed that transcription factor WT1 plays important role in many pathophysiologic processes such as organs development, tumorigenesis and cells proliferation. However, the role of WT1 in PV is still remain unclear. In this study, we will investigate the role of WT1 in the pathogenesis of lesion formation in PV.

Methods Skin specimens and peripheral blood mononuclear cells (PBMCs) were obtained from 25 patients with PV and
20 age- and sex-matched healthy subjects. mRNA and protein levels were detected by real-time RT-PCR and western blot. WT1 siRNA and WT1 overexpression plasmid were transfected into HaCaT cells with lipofectamine 2000 respectively. The proliferation and apoptosis of $\mathrm{HaCaT}$ cells were detected by CCK8 kit and Annexin V-FITC/PI Apoptosis Detection Kit

Results Compared with normal controls, both the mRNA and protein level of WT1 were increased significantly in psoriatic skin and PBMCs. Transfect with WT1 siRNA inhibited the proliferation of $\mathrm{HaCaT}$ cells and promoted $\mathrm{HaCaT}$ cells apoptosis, while WT1 overexpression plasmid exhibited the opposite effects on HaCaT cells. The global DNA methylation level of psoriatic skins and PBMCs were elevated accompanied with increased DNMT1 expression. In addition, an positive correlation was observed between WT1 and DNMT1.

Conclusions Increased WT1 promotes the keratinocytes proliferation and inhibites the apoptosis of keratinocytes which may mediated by recruiting DNMT1 to its target genes related to cell proliferation and apoptotic pathway.

\section{FAMILIALITY AND CONCORDANCE OF PRESENTING MANIFESTATIONS AMONG FILIPINO PATIENTS FROM LUPUS MULTIPLEX FAMILIES}

V Santos*, ST Navarra. University of Santo Tomas Hospital, Internal Medicine - Section of Rheumatology, Manila, Philippines

\subsection{6/lupus-2017-000215.311}

Background and aims To describe the familiality and concordance of manifestations among Filipino patients with systemic lupus erythematosus (SLE) and their affected first degree relatives (FDR).

Methods Filipino mulitplex SLE families with at least 2 first degree relatives (FDRs) diagnosed as SLE were identified from University of Santo Tomas (UST) Lupus Database. Demographic and disease characteristics were described, and types of relationships within families were analysed for concordance of presenting manifestations using McNemar and Fisher's Exact tests, with significance at $\mathrm{p}<0.05$.

Results The prevalence of familial SLE in the UST Lupus Database $(n=2474)$ was $7.8 \%$. There were 192 patients (173, 90\% females) from 95 families (2 families with 3 FDRs), including 25 parent-offspring pairs (23 mother-daughter) and 70 sibling pairs (56 sister-sister). Average age at SLE diagnosis was 31+11.4 SD (range 5-68) years for all affected FDRs. Among parent-offspring pairs, parents' age averaged $44.8+9.7$ SD (range 29-68) years and their offsprings averaged 23.6 +10.6 SD (range 5-35) years at SLE diagnosis, $\mathrm{p}<0.001$. Average age at SLE diagnosis among sibling pairs was 28.6 +11.4 SD (range 9-55) years, with a positive linear association of age at SLE diagnosis between siblings, $p<0.001$. Most common presenting manifestations were malar rash (47\%), oral ulcers (45\%), photosensitivity (40\%), hematologic (39.9\%) and arthritis (39\%). Concordance among related FDRs was significant for oral ulcers and hematologic manifestations, $\mathrm{p}<0.05$.

Conclusions This study underscores the role of genetics in age onset and clinical expression of lupus. 